\begin{tabular}{|l|l|l||}
\hline \multicolumn{2}{|c|}{ PublisherInfo } \\
\hline \hline PublisherName & $:$ & BioMed Central \\
\hline \hline PublisherLocation & $:$ & London \\
\hline \hline PublisherImprintName & $:$ & BioMed Central \\
\hline \hline
\end{tabular}

\title{
6th Nottingham International Breast Cancer Conference (22-24 September 1999)
}

\begin{tabular}{||l|l|l||}
\hline \multicolumn{2}{|c||}{ ArticleInfo } \\
\hline \hline ArticleID & $:$ & 33 \\
\hline \hline ArticleDOI & $:$ & $10.1186 /$ bcr33 \\
\hline \hline ArticleCitationID & $:$ & E002 \\
\hline \hline ArticleSequenceNumber & $:$ & 15 \\
\hline \hline ArticleCategory & $:$ & Meeting report \\
\hline \hline ArticleFirstPage & $:$ & 1 \\
\hline \hline ArticleLastPage & $:$ & 4 \\
\hline \hline & & RegistrationDate : 1999-10-20 \\
ArticleHistory & $:$ & OnlineDate $\quad$ 1999-10-20 \\
\hline \hline ArticleCopyright & $:$ & Current Science Ltd1999 \\
\hline \hline ArticleGrants & $:$ & \\
\hline \hline ArticleContext & $:$ & 130582211 \\
\hline \hline
\end{tabular}


Richard De Boer, ${ }^{\text {Aff1 }}$

Aff1 Department of Medicine, Royal Marsden Hospital, Fulham

Road, London SW3 6JJ, UK

\section{Introduction}

The bi-annual Nottingham International Breast Cancer Meetings are centered upon the presentation of submitted short papers on key areas of breast cancer-related clinical work and research. In addition, there are five invited lectures and a large number of workshops and poster sessions. The fundamental aim is to be an inclusive meeting with significant discussion and participation by most delegates.

At this year's meeting, the five invited lectures were:

1) HER2: a new target for therapy in breast cancer: Dennis Slamon (Los Angeles, USA)

2) The biology of endocrine response in breast cancer - influence of growth factors: Robert Nicholson (Cardiff, UK)

3) Critical review of prognostic and predictive factors: Ian Ellis (Nottingham, UK)

4) Breast cancer in Europe: Umberto Veronesi (Milan, Italy)

5) Optimising local control in breast cancer: Cocq van de Velde (Leiden, The Netherlands)

In addition, major plenary sessions were held on breast screening, role of post-operative radiotherapy, endocrine therapies, chemotherapy and dose intensity, ovarian suppression and ductal carcinoma in situ.

\section{Emerging therapies}

The taxane agents have been used in breast cancer therapy for about a decade. Despite much clinical research, their most appropriate place amongst the numerous chemotherapy agents available remains arguable. JM Nabholtz (Edmonton, Canada) presented data on behalf of the TAX306 study group from a phase III trial comparing the combination of doxorubicin plus docetaxel (AT) versus the standard combination of doxorubicin plus cyclophosphamide (AC) as first line chemotherapy in patients with metastatic breast cancer. Although the data were preliminary, with a short overall median follow-up, the study showed that the AT combination had a significantly higher response rate $(60$ versus $47 \%, \mathrm{P}=$ 0.008 ) as well as having fewer patients progressing whilst on treatment. However, this improved response rate was at a cost of increased neutropaenia (82 versus 69\%), but cardiac toxicity was similar between the groups. Most interesting were the excellent response rates seen in patients with poorprognostic disease. If more mature results indicate a significant survival benefit then the combination of 
a taxane with an anthracycline may become the new first-line standard of care in metastatic breast cancer.

In theory, liposome-encapsulated anthracyclines should have an improved therapeutic index, the active drug being released at the site of tumour. AT Chan (Hong Kong - on behalf of the TLC D-99 study group) presented data from a study comparing liposome-encapsulated doxorubicin (TLC D-99) plus cyclophosphamide versus epirubicin plus cyclophosphamide in the first-line treatment of metastatic breast cancer. The results showed that while response rates were similar (46 versus 39\%), the patients receiving TLC had an improved progression free survival (PFS) of 7.7 versus 6.0 months $(\mathrm{P}=0.04)$. There was however increased toxicity, especially stomatitis, in the TLC group. What impact this study will have on the further development and use of liposome-encapsulated anthracyclines is difficult to know. Although significant, the improvement in PFS is small. Comparisons of toxicity are difficult, as it has been clearly shown that at equimolar doses (as used in this study: $75 \mathrm{mg} / \mathrm{m}^{2}$ ), epirubicin is significantly less toxic than doxorubicin. Finally, a small study was presented by JA O'Hagan (Liverpool, UK) comparing capecitabine with paclitaxel in women with metastatic breast cancer failing previous anthracycline therapy. Capecitibine is a tumour selective oral fluoropyrimidine that is ultimately converted to 5 -fluorouracil $(5 \mathrm{FU})$. Its great potential advantage is its oral route of administration. Results showed that capecitabine had comparable efficacy to paclitaxel but with an improved toxicity profile. Unfortunately, the numbers in the study $(n=42)$ preclude any meaningful conclusions. Nevertheless, further study of this agent is definitely warranted and is proceeding.

\section{Endocrine strategies}

The aromatase inhibitors (AIs) are now the recommended treatment for postmenopausal patients with advanced breast cancer progressing on tamoxifen. A major unanswered question is whether the AIs could be used instead of tamoxifen as first-line therapy. The results of two studies aiming to answer this question were presented by B Thuerlimann (Edmonton, Canada) on behalf of the Arimidex study group. Both studies compared $1 \mathrm{mg}$ anastrozole (arimidex) with $20 \mathrm{mg}$ tamoxifen daily in women eligible for hormone therapy (receptor positive/unknown). Both studies demonstrated that anastozole has equivalent efficacy to tamoxifen and that the two agents have very similar toxicity profiles. While these studies suggest that anastrozole can be seen as an alternative to tamoxifen, tamoxifen, with its long track record and proven efficacy over many years and many studies, remains the first choice for front line hormone treatment in metastatic breast cancer.

On the final morning of the meeting a major session was held, to examine the role of ovarian ablation in the management of premenopausal patients. Four papers were presented (from the USA, Italy, Austria and the UK). Although definite conclusions were difficult to draw, there is growing evidence that ovarian ablation/suppression using the LH-RH agonists may have a significant role to play in the treatment of premenopausal women with oestrogen-receptor-positive disease. The session was provocatively led by the Chairman, Roger Blamey, who presented the results of his interpretation of recent breast cancer treatment overviews of adjuvant chemotherapy and of ovarian ablation. He concluded that the majority of the benefit seen with chemotherapy in premenopausal women was via its effect on the ovary. This led to an interesting discussion amongst the invited speakers to say the least! 


\section{Ductal carcinoma in situ (DCIS)}

The final session of the meeting concerned this disease which has become increasingly important since the advent of mammographic screening and now represents 20-30\% of all new breast cancer cases. The most appropriate form of treatment remains controversial. Is surgery with adequate margins of treatment enough (and if so, what margin is adequate?), or should radiotherapy and/or tamoxifen also be given? Can we predict good- and poor-prognostic subgroups where either less or more treatment is required?

The session saw data presented by the European Organisation for Research and Treatment of Cancer (EORTC) which supports the results of the previous National Surgical Adjuvant Breast Project (NSABP) B-17 study, namely that the addition of radiotherapy to complete local excision of DCIS leads to a decreased incidence of local recurrence. Analysis of the histological subtypes in this study show that there is no correlation between well-, intermediate-, and poorly-differentiated DCIS and the local recurrence rate, but that patients with poorly differentiated DCIS do have a significantly higher incidence of distant metastases. This may therefore point to a group of DCIS patients who may benefit from further treatment.

\section{Conclusion}

Overall, this was an excellent meeting, with a full coverage of the many and varied aspects of breast cancer research and management. Although no significant new breakthroughs were presented here, there certainly is the feeling that steady progress is being made in developing more effective therapies for patients with breast cancer. 